\title{
Use of Gas-Sensor Array Technology in Lung Cancer Diagnosis
}

\author{
Young Jun Kim, Han Young Yu, In-Bok Baek, Chang-Geun Ahn, Bong Kuk Lee, Yarkyeon Kim, \\ Yong Sun Yoon, Ji Eun Lim, Byeong-Jun Lee, Won Ik Jang, Jeong Ho Park, and Chang-Auck Choi
}

\begin{abstract}
Gas-sensor array technology, which has been much utilized in the field of food technology by the name of 'electronic nose' is drawing attention in diagnosing lung cancer based on the analysis of the exhaled human breath. Much understanding has been accomplished about the composition of the volatile organic compounds (VOCs) of the human exhaled breath, in spite of some variations depending on research groups due mainly to lack of the standardization of the sensing procedures. Since VOCs may be produced during the process of cellular metabolism, difference in the cellular metabolism between healthy cells and lung cancer cells are expected to be reflected on the composition variation of the exhaled VOCs. Several studies have attempted to apply the gas-sensor array technology to lung cancer analysis using many different types of sensors including metal oxide, carbon black-polymer composite, surface acoustic wave, and gold nanoparticles. In this mini-review VOC as biomarkers, sensor array technology and application of the array technology for the diagnosis of cancer disease have been described.
\end{abstract}

Keywords : Gas sensors, Sensor array technology, Electronic nose, VOC, Exhaled breath, Lung cancer, Review

\section{INTRODUCTION}

Sensor array technology, in which multiple sensors with different sensing properties are used, was developed with the aim of emulating the functional capability of the biological nose system [1-3]. In real circumstances, the target analyte system is composed not of a single compound but of many different compounds. The limitation of a single sensor application under real circumstances prompted mimicking the natural gas-sensing system of the biological nose system. The basic concept of the sensor array technology is the use of many different types of sensors which possess different sensing behaviors. When those multiple sensors 'sense' the target analyte system of multi-component compounds, a type of 'finger print' pattern is produced. Although the concept of the electronic nose is elegant, producing a meaningful result using the array technology is not such a simple task.

Electronics and Telecommunications Research Institute (ETRI) 218 Gajeong-ro, Yuseong-gu, Dajeon, 305-700, Korea

${ }^{+}$Corresponding author: junkim@etri.re.kr

(Received : Jul. 3, 2013, Accepted: Jul. 16, 2013)

This is an Open Access article distributed under the terms of the Creative Commons Attribution Non-Commercial License(http://creativecommons.org/licenses/by$\mathrm{nc} / 3.0$ )which permits unrestricted non-commercial use, distribution, and reproduction in any medium, provided the original work is properly cited.
Developing the technology, including choice of the sensor element, number of the sensors to be used, and the type of algorithms to be utilized, is very complex. However, the studies investigating the practical applications of the array technology have facilitated its applications in food technology. The development of the gas-sensor array technology is recently finding yet new application in the medical field.

Lung cancer is the major cause of cancer fatality exceeding the total number of death of the next four major cancers, causing more than one million deaths a year around the world [4-6]. The high mortality of the lung cancer may be attributed to the difficulty in early detection of the disease. It is insightful to compare the rate of early detection of the lung cancer (15\%) with that of the overall five-year survival (16\%). The major problem of the lung cancer is that no appreciable symptoms are involved with the early stage of the lung cancer. A series of the highly sophisticated detecting methods have been developed including x-ray, computed tomography (CT), magnetic resonance imaging (MRI) and positron emission tomography (PET). However use of the highly sophisticated technologies has not been successful enough to decrease the number of lung-cancer mortality to any significant degree. Moreover the image-based screening tests require invasive process in which the suspected cells 
are taken out for further study under microscopy. The complication associated with the image-based technologies is also reported to be a disadvantage. High cost, complication, and complex procedure are the major problems of these current methods. A new method which detects VOCs of the cancer patients is drawing much attention with potential advantages over those problems.

GC-MS has been major analytical method of the early studies for correlating exhaled VOCs to lung cancer [7-9]. Thanks to the VOCs studies of the lung cancer a series of sensor array technologies have followed using different types of sensor systems including quartz crystal microbalance [10], metal oxide [11], polymer-carbon black composite [12], surface acoustic wave [13], colorimetric system [14] and gold nanoparticles [15]. Noninvasiveness and low cost of the VOC analysis method seem to be the major driving force for the increasing number of studies in recent years. These studies well support the potential applicability of the gas-sensor array technology in diagnosis of cancer patients through exhaled VOCs. However the compatibility among these studies is very limited due to difficulties in standardization.

For example no standard VOCs for lung cancer patients have been established. Diet, age, smoking and gender may be the sources for the differences in VOCs. Also stages of lung cancer can affect differently for the composition of the VOCs. In order to increase the accuracy of the effect of the VOCs composition on lung cancer, many research groups are trying to be careful about describing age, sex, history of smoking, stages of lung cancer, and surgical history related with lung cancer in the individuals. The variances of the VOCs results may also come from collecting process of the exhaled VOCs, detecting sensor systems and data analysis process for pattern recognition. However in spite of all these complexities many researches are being carried out in the hope of establishing gas-sensor array systems which can analyze the VOCs of the lung cancer patients with high accuracy.

\section{VOC APPROACH FOR LUNG CANCER DETECTION}

\subsection{VOCs and Lung Cancer: Background of VOCs as a Biomarker for Lung Cancer}

VOCs are volatile chemical species that can evaporate relatively easily due mostly to small molecular weight. The volatility is the basis for the possibility of detecting lung cancer based on VOCs from human breath. Indeed human breath has been known to contain more than 1000 VOCs with their concentration ranging from a few parts per trillion (ppt) to parts per million (ppm). The VOCs from the exhaled breath can be traced to either endogenous or exogenous chemicals. Endogenous VOCs are the natural chemical species produced in the human body through various processes of biochemical metabolism. Exogenous VOCs can be traced to the environment around which people reside or travel. The exogenous VOCs can enter the human body through respiration and contact with skin. Since these endogenous and exogenous VOCs circulate around human body through blood stream, it is a reasonable to consider that the VOCs can reflect the condition of a human body. Numerous studies have shown correlation between VOCs from the exhaled breath and lung cancer [7-15]. Most of these VOC analyses have been carried out using gas chromatography mass spectroscopy (GC-MS) and proton transfer reaction mass spectrometry (PTR-MS). The VOCs from human breath contain hydrocarbons, alcohols, aldehydes, and ketones with very small number of esters and nitriles. An excellent review on the VOCs related with lung cancer was written by Hakim et al [16]. Generally higher concentration of VOCs is detected in the exhaled breath of the lung cancer patients than that of the healthy individuals. Oxidative stress is the main cause of variation in the hydrocarbons from the human breath. Lipid peroxidation, which is initiated by removal of allylic hydrogen through reactive oxygen species with final products of alkanes, is the main cause of the alkane production. Isoprene, which is presents in highest concentration among the breath VOCs, is known to vary depending on age and gender [17].

\subsection{Gas-Sensor Array Technology}

Gas-sensor array technology has been developed in an effort to analyze and recognize a target analyte system which is composed of multiple components [18]. Array technology in gas sensors was developed in the name of electronic nose in order to mimic the mammalian nose system. The major driving force for the advent of the array technology is that a single sensor cannot be utilized in a real environment where many different types of gas molecules affect the 'scent'. With more than $10^{7}$ of 
olfactory neurons the mammalian nose is a highly sophisticated sensing system which had been developed through the long evolution history [19]. The multiple sensor elements in the biological system make it, in principle, possible to recognize any analyte system, which is composed of many different types of analyte elements with varying concentrations. Hardly any analyte system is composed of a single analyte element in a real environment. The mammalian nose, with multiple number of sensing elements, does the recognition process through pattern recognition. Those sensing elements have different sensing properties with some degree of overlapping, which enables pattern recognition.

One important concept to understand is the 'crossreactivity'. In the biological nose system a group of olfactory neurons may show a similar sensing behavior toward a certain analyte. However those sensing elements in the group do not necessarily show similar sensing properties toward other types of analytes. Based on the information about the pattern recognition mechanism of the biological nose, the man-made nose system, the electronic nose, adopts the pattern recognition toward multiplecomponent analyte system through the use of multiple sensor elements. The beauty of the electronic nose is that each constituting sensor element doesn't need to equip ideal selectivity [20].

The invention of the electronic nose had been prompted because of the limit of the sensing properties of the manmade gas sensors. The metal oxide sensor, which is the most popular commercial product with the longest history in sensors, shows very poor capability in selectivity. Many methods have been attempted in an effort to provide selectivity, including doping the semiconductors with noble metal catalysts [21], modifying the operational temperature, varying the particle size and porous structure of the semiconductor metal oxide [22] and using selective membrane [23]. However obtaining satisfying degree of selectivity is very difficult. However with the use of concept of electronic nose, array of metal oxide sensors can be applied for real system such as food technology [24].

Many different types of gas sensors have been utilized for electronic nose system. Metal oxides are the most widely used materials for gas sensor application. Metal oxide materials used for sensor application include $\mathrm{SnO}_{2}$, $\mathrm{TiO}_{2}, \mathrm{WO}_{3}, \mathrm{~V}_{2} \mathrm{O}_{3}, \mathrm{Fe}_{2} \mathrm{O}_{3}, \mathrm{Mn}_{2} \mathrm{O}_{3}$ etc. In the metal oxide gas sensors the atmospheric oxygen is in equilibrium with the surface of the metal oxide sensor. When an analyte molecule comes in contact with the metal oxide surface, redox reaction occurs. The redox reaction on the sensor surface is observed as a change in the resistance of the sensor materials. The magnitude of the resistance change is observed as the amount of the gas molecules detected. Accordingly the magnitude of resistance change can be correlated with the concentration of the target analyte. However the redox reaction between the metal oxide surface and the incoming analyte molecules does not occur at room temperature. In order for the redox reaction has to occur the barrier to the redox reaction has to be overcome by increasing temperature $\left(300 \sim 450^{\circ} \mathrm{C}\right)$. That is why the metal oxide sensor has to be equipped with power source [25].

Conductive materials are also used for gas sensors in the form of composite with insulating materials. Conductive polymers (polypyrrole, polyaniline and polythiophine), metal particles and carbon can be used for conducting materials. Diverse types of polymers may be used as insulating materials. The composite sensor system can be envisioned as domains of conductive materials embedded in the matrix of insulating materials. Transducer is formed when the composite material is positioned between electrodes. When analyte molecules come in contact with the sensor composite surface, the analyte molecules are absorbed by the insulating polymer matrix. In most of the composite sensors, the interaction of the sensors with the analytes are observed as increase in resistance. The increase in resistance of the sensor is interpreted as increased distance between the conductive materials embedded in the insulating matrix due to swelling of the matrix polymer by the absorbed analytes [26].

The composite system with polymers has some advantages over the traditional metal oxide sensors. Different from the metal oxide sensors, in which high temperature is required for sensing, the polymer composite system does not require increased temperature for detecting procedure. Another major advantage is selectivity. In the composite system selectivity can be easily varied by simply using different types of polymers. The ease of diversifying selectivity is based on the fact that the interaction of the incoming analyte molecule with the sensor is dominated by the types of insulting polymer matrix. Availability of many different types of commercial polymers is another advantage. Since the composite sensor system does not need power source, application to portable sensor is another advantage of the composite sensor. Availability of 
such diverse types of sensors may have contributed to the advent of electronic nose system in which many different types of sensor element are required. Food analysis is an area where electronic nose can contribute a lot. Since aroma from food also is a mixture of different molecules, pattern recognition using sensor array can be understood to be an apt application. In deed electronic nose has been increasingly used in food industry [27]. Food industry is traditionally a proper application field for electronic nose. Recently the sensor-array technology is finding yet new application in medical field.

\subsection{Application of the Array Technology in the Analysis of Lung Cancer}

Use of sensor array technology for lung cancer analysis may seem to be a reasonable approach. Human breath contains multiple VOCs, and the difference exists in the VOCs composition between the cancer patients and healthy individuals. Many different types of gas-sensor array systems have been used for applications in the lung cancer diagnosis [10-15].

One of the earliest studies for identification of the exhaled breath of the lung cancer patients was carried out by Gordon et al [7]. Using GC-MS exhaled VOCs of the lung cancer patients (12 of lung cancer patients and 17 control samples) were identified. The sample VOCs were collected by exhaling into a sampling bag. Preconcentration was carried out using the Tenax GC sorbent cartridge. Much recognizable peaks were observed at the relative retention index at 659,1095 and 1248 with the \% occurrence of lung cancer against control, 50.0 to $0.0,50.0$ to 0.0 and 75.0 to 0.0 . This study well provided relevancy of the correlation between VOCs and lung cancer. It is noteworthy that this study described the significance of the normalization of samples donors based on age and gender. Many other groups also used GC-MS for evaluation of the VOCs in lung cancer patients [9, 28, 29].

Sensor array technology based on quartz microbalance (QMB) was used by Natale et al [10]. Eight different types of metalloporphyrins were used as the coating materials. These sensors were confirmed to have shown good response towards aromatic compounds, amines, alcohols and ketones, which had been known to be possible breath biomarkers for lung cancer. 60 individuals were involved in the test (35 of them are lung cancer patients, 16 were healthy individuals for control and 9 individuals had gone through a surgical therapy). Based on partial least squaresdiscriminant analysis the cancer patients were classified $100 \%$ and the reference group $94 \%$. It is interesting to take note of the post-surgery groups, which resulted in $44 \%$ while the rest of the post-surgery patients were confirmed to be healthy. The people under the examination all went through the same diet and the same mouth hygiene.

Chen et al [13] used an array of surface acoustic wave (SAW) sensors to demonstrate that the SAW assay is an effective method for the recognition of the lung cancer patients. One noteworthy set for this system is the use of GC capillary column. The GC column was connected between a gas injector and a SAW sensor array. GC column separates those VOCs injected for the test. The analysis system includes solid phased micro extraction (SPME) and a capillary column for pre-concentration. Polyisobutylene (PIB) was used as a coating material for the SAW system. Artificial neural network system has been used for recognition process. Eleven hydrocarbon VOCs previously confirmed to be lung cancer biomarkers, were used to check for the eligibility of the sensor system. The eleven lung cancer VOCs included four aromatic VOCs (benzene, styrene, propyl benzene and 1, 2, 4trimethyl benzene), three saturated hydrocarbon VOCs (decane, undecane and methyl cyclopentane), two unsaturated hydrocarbon VOCs (isoprene and 1-hezene) and two aldehyde VOCs (hexanal and heptanal).

Machado et al used commercially available electronic nose to discern lung cancer patients from healthy people [12]. The commercial electronic nose used in this study is 'Cyranose 320' which contain 32 different polymer-carbon black composite. The working principle of the polymercarbon black composite sensor can be envisioned as the conducting carbon black dispersed in the insulator polymer matrix. When the analyte molecules come in contact with the composite sensor film, the composite film swells, which results in increase in resistance of the sensor film due to increase in the distance between conducting carbon black in the insulating polymer matrix. Exhaled breath of 14 individuals with bronchogenic carcinoma and that of 45 healthy reference individuals were analyzed. The results based on the principle component and canonic discriminant analysis support the discrimination capability between lung cancer patients and healthy individuals. However this report also mentioned that further study would have to be carried out with less-advanced patients since the patients under this study were all advanced with lung cancer. 
Mazzone et al used a colorimetric sensor array comprised of 36 different chemically sensitive materials in an effort to diagnose lung cancer [14]. The sensor materials were assorted into five categories, metalloporphyrins, acid indicators, base indicators, vaporchromic and metal salts. These indicator chemicals were impregnated into a polyethylene terephthalate (PET) film. Three groups of people, those with lung cancer, those with other types of lung disorder and healthy people, were involved in the test. The test individuals were placed in a room for 12 minutes of breathing for normalization. Total 143 individuals were involved: 49 with non-small cell lung cancer, 18 with chronic obstructive pulmonary disease, 15 with idiopathic pulmonary fibrosis, 20 with pulmonary arterial hypertension 29 with sarcoidosis and 21 controls. The colorimetric sensor array used in this study was shown to produce unique VOC patterns for the lung cancer patients with moderate accuracy. Barash and Haick proposed a possibility of using gold nanoparticle gas sensors for classifying lung cancer histology [15]. Diverse types of gold nanoparticles had been used for gas sensor applications [21, 22]. Gold nanoparticles used in this study may form sensor film on interdigitated electrode through spray coating. The working principle of the gold nanoparticle sensors can be understood from the structural feature of the gold nanoparticle. The gold nanoparticle is composed of inner gold core encapsulated with organic ligand molecules. The gold nanoparticle film can be envisioned as an insulating film of organic matrix embedded with conducting gold spheres, which, when in contact with analyte molecules, swells resulting in increase in resistance. In this study gold nanoparticle sensors which had been confirmed by GC-MS to be qualified for analysis of the VOCs for lung cancer were used for the study, which involved headspace analysis of the lung cancer cell. Particular point to take note of is that the sample cells were not simply categorized into the healthy and the diseased. The lung cancer cells were further compared between small cell lung cancer and non-small cell lung cancer, and between two subtypes of the non-small cell lung cancer, adenocarcinoma and squamous cell carcinoma. The analysis of the VOCs was able to differentiate between the healthy and lung cancer cells, between non-small cell lung cancer and small cell lung cancer. The results demonstrated even the subtypes of non-small cell lung cancer.

\section{CONCLUSIONS}

Although it may be beyond the scope of this mini-review to describe all the aspects of the studies related with lung cancer diagnosis by the exhaled human breath, we have at least tried to summarize as many aspects as possible in this brief review by including each group's research studies with emphasis on normalization, sensor systems, VOCs interested in the study and the importance of the results when necessary. Even with complexities involved with standardization and normalization in the test individuals, procedure for collecting exhaled VOCs, sensing systems and pattern recognition process, most studies produced results that support significance of the exhaled VOC application for lung cancer diagnosis. Recently researches are oriented toward more specific diagnosis of the lung cancer in order to specify the stages of the lung cancer. With more establishment in the above mentioned difficulties involved with normalization and standardization, the application of the gas-sensor array technology for the screening and diagnosis of the lung cancer is thought be realized in the near future.

\section{REFERENCES}

[1] F. Rock, N. Barsan, and U. Weimar, "Electronic nose: current status and future trends", Chem. Rev., Vol. 108, No. 2, pp. 705-725, 2008.

[2] M. Peris and L. Escuder-Gilabert, "A 21st century technique for food control: electronic noses", Anal. Chim. Acta., Vol. 638, No. 1, pp. 1-15, 2009.

[3] H. Smyth and D. Cozzolino, "Instrumental methods (spectroscopy, electronic nose, and tongue) as tools to predict taste and aroma in beverages: advantages and limitations", Chem. Rev., Vol. 113, No. 3, pp. 14291440, 2013.

[4] A. Jemal, R. Siegel, E. Ward, Y. Hao, J. Xu, M. J. Thun, "Cancer statistics, 2009", CA-Cancer J. Clin., Vol. 59, No. 4, pp. 225-249, 2009.

[5] A. Jemal, R. Siegel, J. Xu, and E. Ward, "Cancer statistics, 2010”, CA-Cancer J. Clin., Vol. 60, No. 5, pp. 277-300, 2010.

[6] D. M. Cutler, "Are we finally winning the war on cancer”, J. Eco. Perspect., Vol. 22, No. 4, pp. 3-26, 2008.

[7] S. M. Gordon, J. P. Szidon, B. K. Krotoszynski, R. D. 
Gibbons, and H. J. O'Neill, "Volatile organic compounds in exhaled air from patients with lung cancer", Clin. Chem., Vol. 31, No. 8, pp. 1278-1282, 1985.

[8] H. J. O’Neil., S. M., Gordon, M. H. O’Neil, R. D. Gibbons and J. P. Szidon, "A computerized classification technique for screening for the presence of breath biomarkers in lung cancer", Clin. Chem., Vol. 34, No. 8, pp. 1613-618, 1988.

[9] G. Preti, J. N. Labows, J. G. Kostelc, S. Aldinger, and R. Daniele, "Analysis of lung air from patients with bronchogenic carcinoma and controls using gas chromatography-mass spectrometry", J. Chromatogr. B, Vol. 432, pp. 1-11, 1988.

[10] C. D. Natale, A. Macagnano, E. Martinelli, R. Paolesse, G. D. Arcangelo, C. Roscioni, A. F. Agro, and A. D. Amico, "Lung cancer identification by the analysis of breath by means of an array of nonselective gas sensors", Biosens. Bioelectron., Vol. 18, No. 10, pp. 1209-1218, 2003.

[11] J. B. Yu, H. G. Byun, J. O. Lim, and J. S. Huh, "Exhaled breath analysis of lung cancer patients using metal oxide sensor", 2011 First ACIS/JNU International Conference on Computers, Networks, Systems, and Industrial Engineering, No. 5954327, pp. 281-284, 2011.

[12] R. F. Machado, D. Laskowski, O. Deffenderfer, T. Burch, S. Zheng, P. J. Mazzone, T. Mekhall, C. Jennings, J. K. Stoller, J. Pyle, J. Duncan, R. A. Dweik, and S. C. Erzurum, "Detection of lung cancer by sensor array analyses of exhaled breath", Am. J. Resp. Crit. Care., Vol. 171, No. 11, pp. 1286-1291, 2005.

[13] X. Chen, M. Cao, Y. Li, W. Hu, P. Wang, K. Ying, and H. Pan, "A study of an electronic nose for detection of lung cancer based on a virtual SAW gas sensors array and imaging recognition method", Meas. Sci. Technol., Vol. 16, No. 8, pp. 1535-1546, 2005.

[14] P. J. Mazzone, J. Hammel, R. Dweik, J. Na, C. Czich, D. Laskowski, and T. Mekhail, "Diagnosis of lung cancer by the analysis of exhaled breath with a colorimetric sensor array", Thorax, Vol. 62, No. 7, pp. 565-568, 2007.

[15] O. Barash, N. Peled, U. Tisch, P. A. Bunn, F. R. Hirsch, and H. Haick, "Classification of lung cancer histology by gold nanoparticle sensors", Nanomedicine: NBM, Vol. 8, No. 5, pp. 580-589,
2012.

[16] M. Hakim, Y. Y. Broza, O. Barash, N. Peled, M. Phillips, A. Amann, and H. Haick, "Volatile organic compounds of lung cancer and possible biochemical pathways", Chem. Rev., Vol. 112, No. 11, pp. 59495966, 2012.

[17] W. Miekisch, J. K. Schubert, and G. F. E. N.Schomburg, "Diagnostic potential of breath analysisFocus on volatile organic compounds", Clin. Chim. Acta, Vol. 347, No. 1-2, pp. 25-39, 2004.

[18] F. Rock, N. Barsan, and U. Weimar, "Electronic nose: Current status and future trends", Chem. Rev., Vol. 108, No. 2, pp. 705-725, 2008.

[19] P. Gostelow, S. A. Parsons, and R. M. Stuetz, "Odour measurements for sewage treatment works", Water Res., Vol. 35, No. 3, pp. 579-597, 2001.

[20] K. J. Albert, N. S. Lewis, C. L. Schauer, G. A. Sotzing, S. E. Stitzel, T. P. Vaid, and D. R. Walt, "Cross-reactive chemical sensor arrays", Chem. Rev., Vol. 100, No. 7, pp. 2595-2626, 2000.

[21] V. Krivetskiy, A. Ponzoni, E. Comini, S. Badalyan, M. Rumyantseva, and A. Gaskov, "Selectivity modification of $\mathrm{SnO}_{2}$-based materials for gas sensor arrays", Electroanal., Vol. 22, No. 23, pp. 2809-2816, 2010.

[22] G. Liu, Z.-J. Tan, C. Wang, R.-F. Wu, and H.-J. Zhang, "Soft template synthesis of high selectivity mesoporous $\mathrm{SnO}_{2}$ gas sensors", J. Shanghai Univ., Vol. 14, No. 4, pp. 297-300, 2010.

[23] J. P. Viricelle, A. Pauly, L. Mazet, J. Brunet, M. Bouvet, C. Varenne, and C. Pijolat, "Selectivity improvement of semi-conducting gas sensors by selective filter for atmospheric pollutants detection", Mater. Sci. Eng. C-Mater. Biol. Appl., Vol. 26, No. 3, pp. 186-195, 2006.

[24] M. G. Varnamkhasti, S. S. Mohtasebi, M. L. R.Mendez, J. Lozano, S. H. Razavi, and H. Ahmadi, "Potential application of electronic nose technology in brewery", Trends Food Sci. Technol., Vol. 22, No. 4, pp. 165-174, 2011.

[25] C. Wang, L. Yin, L. Zhang, D. Xiang, and R. Gao, "Metal oxide gas Sensors: sensitivity and influencing factors", Sensors, Vol. 10, No. 3, pp. 2088-2106, 2010.

[26] H. Lei, W. G. Pitt, L. K. McGrath, and C. K. Ho, "Modeling carbon black/polymer composite sensors," Sens. Actuator B-Chem., Vol. 125, No. 2, pp. 396-407, 
2007.

[27] E. A. Baldwin, J. Bai, A. Plotto, and S. Dea, "Electronic noses and tongues: Applications for the food and pharmaceutical industries", Sensors, Vol. 11, No. 5, pp. 4744-4766, 2011

[28] M. Phillips, K. Gleeson, J. B. M. Hughes, J. Greenberg, R. N. Cataneo, L. Baker, and W. P. McVay "Volatile organic compounds in breath as markers of lung cancer: A cross-sectional study", Lancet, Vol. 353, No. 9168, pp. 1930-1933, 1999.

[29] D. Poli, P. Carbognani, M. Corradi, M. Goldoni, O. Acampa, B. Balbi, L. Bianchi, M. Rusca, and A. Mutti. "Exhaled volatile organic compounds in patients with non-small cell lung cancer: cross sectional and nested short-term follow-up study", Resp. Res., Vol. 6, No. 71, pp. 1-10, 2005.

[30] Y. J. Kim, Y. S. Yang, S. C. Ha, S. M. Cho, Y. S. Kim , H. Y. Kim, H. Yang, and Y. T. Kim, "Mixed-ligand nanoparticles of chlorobenzenethiol and n-octanethiol as chemical sensors", Sens. Actuator B-Chem., Vol. 106, pp. 189-198, 2005.

[31] N. Krasteva, I. Besnard, B. Guse, R. E. Bauer, K. Muller, A. Yasuda, and T. Vossmeyer, "Selfassembled gold nanoparticle/dendrimer composite films for vapor sensing applications", Nano Lett., Vol. 2, No. 5, pp. 551-555, 2002. 